\title{
Dual Measurement Mode Rotational Viscometer
}

\author{
Tapio Fabritius \\ Optoelectronics and Measurement \\ Techniques Research Unit \\ University of Oulu \\ Oulu, Finland \\ tapio.fabritius@oulu.fi
}

\author{
Paivo Kinnunen \\ Particle Engineering Researh Unit \\ University of Oulu \\ Oulu, Finland \\ paivo.kinnunen@oulu.fi
}

\author{
Jakub Czajkowski \\ HoloLens Team \\ Microsoft \\ Espoo, Finland \\ jakub.czajkowski@microsoft.com
}

\begin{abstract}
A novel dual method concept to widen the viscosity measurement range of magnetic actuation and sensing based viscometer is presented. A phase slipping detection is used for high viscosities, while fluids with low viscosities are studied by applying a step-stress and detecting early stage strain oscillations. Developed dual mode concept was tested and verified by studying water-glycerol solutions with different concentrations. The results show that proposed method significantly improves the measurement capability of the rotational viscosity probe without increasing the overall cost of the system or compromising the advantages (non-contact, nonoptical, disposable, hermetic measurements) of it.
\end{abstract}

\section{Keywords-fluid properties, measurement system, rheology}

\section{INTRODUCTION}

Rheometers are used in many fields of research and development to investigate complex material flows and deformation under the applied stress. They can be used for fluids such as emulsions, suspensions, polymers, foams, gels, and micellar solutions [1-3]. Accurate characterization of complex fluids enables rapid development in nanotechnology, biotechnology, medicine, engineering, physics, chemistry, etc. being thus in high interest of rheology research. In the industry, unsteady behavior of complex fluids is of fundamental importance $[4,5,6]$ helping in developing novel processes and optimization of the existing solutions [7, 8]. In biotechnology and medicine researchers relay on rheology studying new drugs and bio-activities [9-15].

The most common rheometers are either synchronous viscometers which are utilizing a motor for driving and a torque sensor, or viscometers which are using a servo motor and a system to determine the rotation speed. Measuring geometries are typically either cone-and-plate or concentric cylinder cell type $[16,17]$. Those classical viscometers require either a physical connection, or a clear optical path and adequate lighting conditions to work properly limiting their applicability. To overcome those limitations, magnetic detection is an interesting alternative for further development.

Our viscometer setup employing simultaneous magnetic actuation and sensing has been published [18]. A permanent ring magnet is attached to the disposable rotor and two pairs of inductor coils act as a stator. The coils are driven by high current to control the actuation of probe and an anisotropic magnetoresistive (AMR) sensor is used for sensing the probe movements. Due to the simplicity of the setup, it is inexpensive. Moreover, it is capable for characterizing nontransparent fluids under a full immersion and the probe can be

This research was carried out as a part of MAGBIO project $(2037 / 31 / 2013)$ and RHEO-DISP (1335/31/2016) funded by the Finnish Funding Agency for Innovation (Tekes/Business Finland). The first author is also partially supported by Academy of Finland's FIRI funding (grant no. 320017) changed or disposed if needed. The sensing method is an extension to the existing rotational rheometers, which means that it can be easily adopted to study anomalous viscosity, viscoelasticity, thixotropy, dilatancy, etc. [19, 20].

In order to analyze the viscosity characteristic liquids, the magnitude [18] or the frequency [20] of the external rotating magnetic field is altered, until a sudden change in the rotational response of a probe happens. This occurs when the rotation mode changes from synchronous to asynchronous, causing a fast back and forward motion of the probe $[12,15$, 21]. That breakdown condition is proportional to the dynamic viscosity of the solution. However, once the inertia of the instrument increases, the accuracy of the measurement decreases for low viscosity solutions [18] limiting the dynamic measurement range of it. The setup was found to provide proper viscosity values from $10 \mathrm{cP}$ to $\sim 400 \mathrm{cP}$ [18].

The inertial effects are often considered problematic, because they limit the ability to measure the theoretical creep response of material at short times [22, 23]. However, it has demonstrated that inertial effects, normally limiting the frequency range accessible by forced oscillations, can be used as advantage [22]. The controlled-stress mode was well known in the area of soft material testing and was particularly useful in carrying out creep tests [24]. Several reports show that inertio-elastic creep ringing can be exploited for the rapid estimation of viscoelastic properties of fluids [5,22,23,25-32] and slightly extend the accessible range of oscillatory measurements.

In this paper, we demonstrate a significant extension of the viscosity sensing capabilities of the rotational viscometer based on simultaneous magnetic actuation and sensing. Inertia of the instrument limiting the dynamic range in phase slipping detection mode [18] is now used in order to determine the viscosity of the solution through a stress step test and analysis of ringing oscillations.

\section{MATERIALS AND MEASUREMENT SETUP}

\section{A. Materials}

Although the proposed method is an extension to the existing rotational viscometer and capable of analyzing complex fluids, the known solutions were used in experimental studies to obtain easily comparable results. The viscosity properties of aqueous solutions with glycerol where tuned by changing the weight of the components ( $\mathrm{w}_{\text {glycerol }} / \mathrm{W}_{\mathrm{water}}$ ). The investigated concentrations ranged from 0 to $95 \%$ of glycerol (Sigma-Aldrich SZBD164CV for the $10-50 \%$ and J. T. Baker 1320405865 for the 60-95\% solutions) were obtained by adding the glycerol into a test 


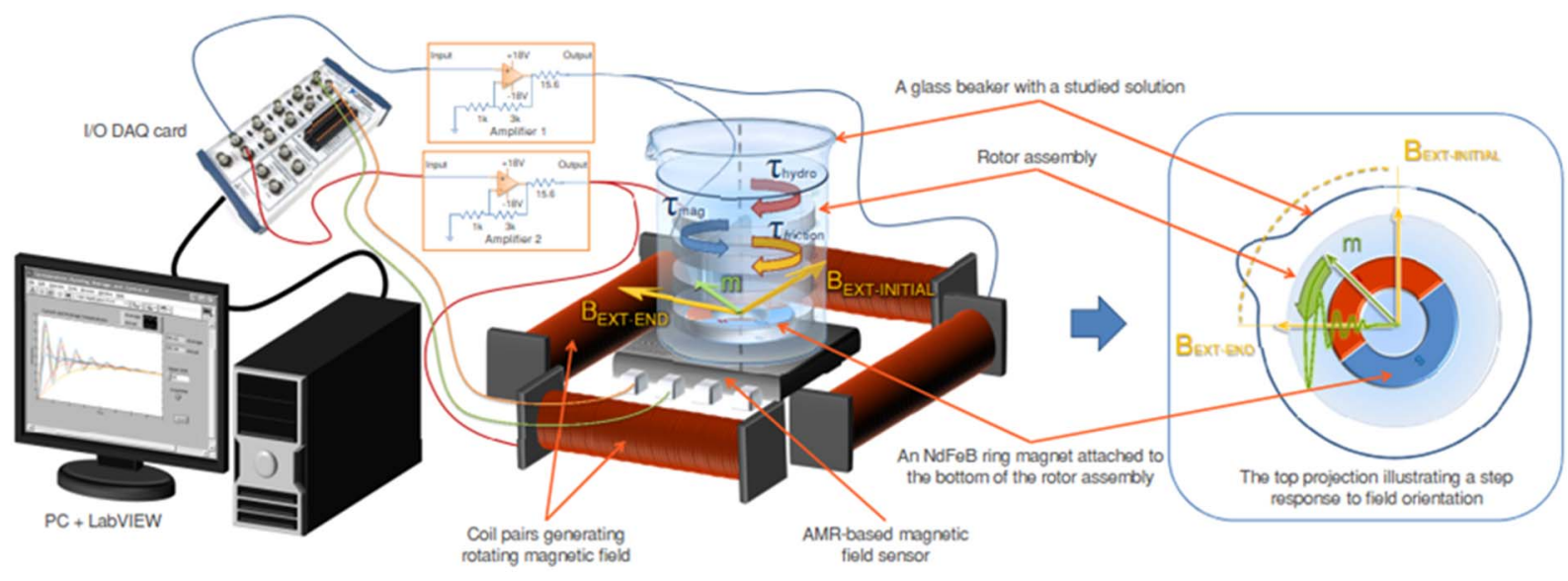

Fig. 1. Used disposable rotational viscometer based on magnetic actuation and sensing. In this context, the disposability means that all parts which are in contact with tested liquid can be disposed.

tube and diluting with ultrapure water. In addition to the water-glycerol measurements, the calibrated liquids (RT5, RT10, RT50, RT100 and RT250) from Paragon Scientific Ltd were used in traceability measurements.

\section{B. Measurement setup}

The used device is a rotational viscometer, where a permanent ring magnet is embedded in a rotor and it is driven by magnetic field. In order to achieve a rotating magnetic field, two-axis, inductor coils (ELC54-19-10000, ERSE Audio) are connected in pairs. Two sinusoidal waves with 90 degrees phase difference are used to drive the coils in phase slipping detection mode. During creep experiments, the orientation of the driving field vector was altered by $100^{\circ}$ by applying constant current to each coil pair. The actuating signals generation is done using a computer with LabVIEW and a DAQ card (PCIe-6363 X Series, National Instruments). and amplified by high-current operational amplifier circuits based on discrete components (OPA548, Texas Instruments). The magnetic field can be controlled for frequency from $0 \mathrm{~Hz}$ to tens of $\mathrm{kHz}$.

The probe consists of four co-planar plates to increase the surface interaction with the fluid and it is positioned in a beaker. A diametrically magnetized $\mathrm{NdFeB}$ ring magnet ( $\mathrm{R}-$ 10-07-03-N, Supermagnete) is mounted below the bottommost disk of the probe. The pin tip on a quartz glass is used as a bearing for the rotor. The angular displacement of the magnet is recorded by AMR sensor (AA747, Sensitec $\mathrm{GmbH})$. The detailed description of the device parameters and angle calculation method is provided in our previous paper [18].

\section{PROPOSED SIGNAL PROCESSING METHODS}

\section{A. Creep ringing method}

A step-stress or "creep" method is based on the measurement of transient response of material to an externally applied step-change. A commonly observed feature of creep experiments on viscoelastic materials is the damped strain oscillations in the early stage of deformation. This phenomenon, known as "creep ringing", results from the coupling of the measuring system's moment of inertia with the sample's (visco)elasticity, providing a prospect for the extraction of the viscoelastic properties of soft materials.
When a step shear stress is applied to a rotational viscometer, the orientation of the external magnetic field is changed and the probe tries to comply with the condition. However, the rapid change of conditions combined with the inertia of instrument cause the probe to overshoot its target orientation and is then pulled back by the elasticity of the studied fluid. As an effect, we observe a series of oscillations which decay with time until the equilibrium state is achieved. During these oscillations, the rotational energy of the probe is transferred into strain energy. When studying the response of a probe, we can observe the inertio-elastic behavior [5, 22, $23,25,27]$. In terms of signal we can observe under-damped sinusoid, which decays exponentially over time as shown in Fig. 2.

The relation between the inertia of instrument and the viscoelastic properties of the studied fluid was defined by Krieger [30] and further explored by others [5, 22, 23, 25, 27, $33]$ In case of the linear theory, the body with viscoelasticity is considered as a linear system. Therefore, the response functions to an excitation $\mathrm{T}(\mathrm{t})$ are known to play a fundamental role both from a mathematical and physical point of view. [34] The equation of motion for the probe, can be written so that:

$$
I \frac{\partial D^{2}}{\partial t^{2}}=\Gamma_{\text {applied }}-\Gamma_{\text {resistant }}
$$

where $I=I_{\text {geometry }}+I_{\text {rheometer }}$ is the inertia, $D$ is the axis angular displacement and $\Gamma_{\text {applied }}$ and $\Gamma_{\text {resistant }}$ are the applied and effective torques, respectively [22]. For linear materials the equation can be rewritten in terms of stress in the following form:

$$
\alpha \frac{\partial \gamma^{2}}{\partial t^{2}}=\sigma_{\text {applied }}-\sigma_{\text {resistant }}
$$

where $\alpha=\frac{I F_{\sigma}}{F_{\gamma}}, \gamma$ is the deformation, $\alpha$ is the inertia parameter, $\sigma_{\text {applied }}$ and $\sigma_{\text {resistant }}$ are respective stresses. $\alpha$ involves the inertia momentum of the mobile part, but also the shear stress and shear rate geometry factors $F_{\sigma}$ and $F_{\gamma}$ of the geometry.

\section{B. Critical amplitude measurement}

In this context, the critical amplitude of current corresponds to the magnitude of the external magnetic field 


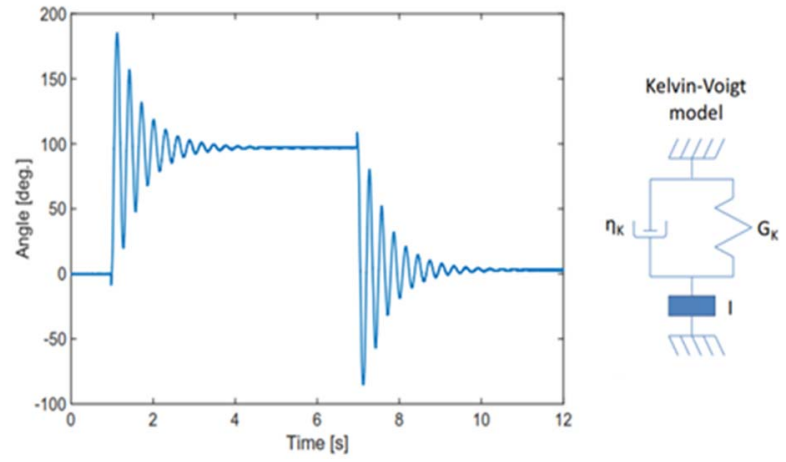

Fig. 2. A stress-step response curve of a pure water (left) and a KelvinVoigt model used in the study (right).

for which oscillation of the rotor changes between synchronous and asynchronous. This sudden breakdown was observed and described by Helgesen et al. [21].

The actuating magnetic field causes a torque on the rotor $\left(\tau_{m a g}\right)$ and the rotor is influenced by a hydrodynamic torque $\left(\tau_{\text {hydro }}\right)$, which works against the magnetic torque (See Fig, 1$)$. Basically, the probe is phase-locked with the field in synchronous rotation mode. However, the probe rotates with the shift of angle $\phi=\Omega-\theta$, where $\Omega$ is the angle of the external magnetic field and $\theta$ is the orientation of a magnet of the rotor. In synchronous mode, the angle $\phi$ depends on the rheology of the solution (and properties of the device). At higher rotation rates, or at low field magnitudes, the probe is not able follow the external field and exhibits a sudden breakdown. In our case that occurs at a critical magnitude of the external magnetic field $B_{c}$. Qualitatively, this phenomenon can be described as the sequence of forward and backward rotations.

\section{RESULTS AND DISCUSSION}

The developed disposable rotational viscometer based on simultaneous magnetic actuation and sensing (Fig. 1) was used to study water-glycerol solutions of different concentrations. Our new approach combines two measurement modes (phase slipping and creep to extend the dynamic range and enable detection at low viscosities. In addition, calibrated liquids were measured in traceability studies. Since the phase slipping method has been investigated in our previous paper [18] this paper is concentrating on the creep method and how it can be exploited together with phase slipping method.

\section{A. Stress-step and inertia-related oscillation}

A ringing oscillation, as shown in Fig. 2, results from a coupling between instrument's inertia and sample's elasticity and can be used to determine the dynamic viscosity of a

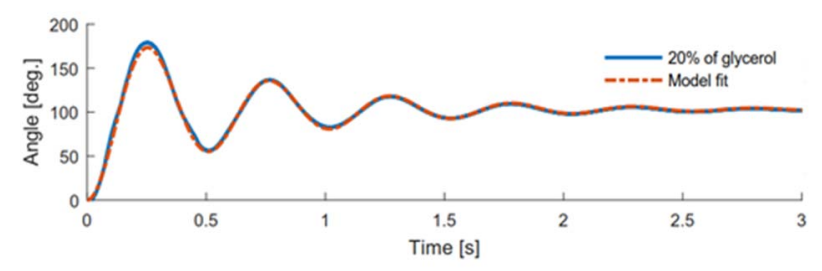

Fig. 3. Kelvin-Voigt model fitting for oscillations experimentally recorded with aqueous solution of $20 \%$ glycerol.

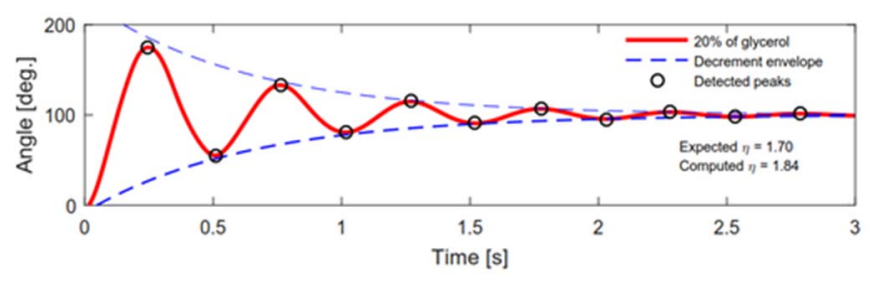

Fig. 4. Peak detection in the oscillation signal recorded with aqueous solution of $20 \%$ glycerol and the result of exponential fitting.

solution. When a step shear stress is applied, the probe tries to comply with the new condition. However, the rapid change combined with instrument's inertia cause the probe to overshoot its target and is then pulled back by the studied fluid, which causes a series of under-damped oscillations.

\section{B. Kelvin-Voigt model fitting}

In order to characterize the properties of the fluid, we chose a linear Kelvin-Voigt model (KV) shown in Fig. 2. The model consists of a Voigt element, i.e., a spring $G_{K}$ and a dashpot $\eta_{K}$ in parallel, connected in series with the inertial term $I$.

For a linear KV model only for an elasticity above the critical value of $G_{K}>\frac{\eta^{2}}{4 \alpha}$ will oscillations occur. The strain can then be defined as a function of time using the following formula:

$$
\begin{aligned}
& \gamma(t)=\gamma\left\{1-e^{-Q t}\left[\cos (\omega t)+\frac{Q}{\omega} \sin (\omega t)\right]\right\} \\
& \text { where } \gamma=\frac{\tau_{0}}{G}, Q=\frac{\eta}{2 \alpha}, \omega=\sqrt{\frac{G}{\alpha}-\left(\frac{\eta}{2 \alpha}\right)^{2}}, \quad \text { so that }
\end{aligned}
$$

coefficient $\alpha$ corresponds proportionally to the geometry factor $\kappa$ and inversely proportional to instrument's inertia $I$ $[22,32]$.

The signals recorded during the measurement for each of the glycerol solutions were loaded into Matlab. Signals were then fitted using the KV model equation. As an example, the obtained results for $20 \%$ glycerol solution is plotted in Fig. 3. We can see a good agreement between the recorded signal and the fitted curve, which confirms the validity of KV model. The slight differences between the profiles could be attributed to friction or wobblyness of the probe.

Fitting the measured data using Eq. 3 allows us to determine the values for a viscous element $\eta$ and for the element $\alpha$, corresponding to the geometry used in the setup and its inertia. The applied stress $\tau_{0}$ is constant throughout the experiments. The obtained coefficients were compared with the theoretical viscosity curve for aqueous glycerol solutions [35]. The dynamic viscosity of a solution was then determined as $\eta_{e f f}=\beta \eta_{K V}^{2}$, where $\beta$ is an empirical calibration constant determined for $50 \%$ glycerol solution. The viscosities determined this way using KV model are plotted in Fig. 5.

\section{Peak detection and exponential decay}

The same data set was processed in a different fashion. After loading the files into Matlab, an algorithm was applied to the measured signal in order to find its local maxima. The results are shown in Fig. 4. We can see that the oscillation results in an underdamped sinusoid, which eventually reaches 


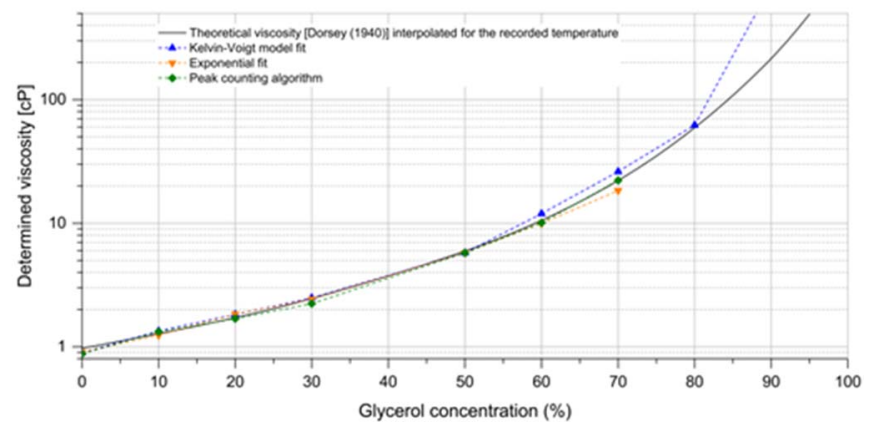

Fig. 5. Viscosities determined from stress-step oscillation tests using various algorithms.

the equilibrium strain value. Consequently, the approximate elastic response can be described as:

$$
J(t)=X e^{-\frac{\Delta \omega}{2 \pi} t} \sin (\omega t+\Psi)+Y
$$

where $\omega$ is ringing frequency, $\psi$ is phase offset, and $Y$ is the bias. In practice, the logarithmic decrement can be determined using absolute value of the peaks in the recorded signal [23] or by applying a regression and fitting the data with an exponential decay [25]. Therefore, the decrement envelope can be described by following formula: $Y(t)=$ $a e^{-b t}$

In order to accurately determine the coefficients, the determined signal peaks were fitted using the envelope formula. The resulting curves are plotted in Fig. 4. Using coefficient $b$ computed during fitting procedure, we are able to determine the dynamic viscosity of glycerol solutions as: $\eta_{\text {eff }}=\alpha b^{2.5}$, where $\alpha$ is a device-related constant and can be determined empirically. The value was determined by comparing computed number with a theoretical viscosity of a $50 \%$ glycerol solution. The viscosities determined this way using exponential fit are plotted in Fig. 5. Moreover, in the same figure we demonstrate that it is possible to determine the viscosity based on the number of recorded oscillations as $\eta_{\text {eff }}=\delta \frac{1}{N^{2}}$, where $\delta$ is empirical calibration constant. Such approach proves to be accurate, while at the same time the method is more tolerant to inconsistent sampling and to signal distortions.

\section{Dual detection mode rheometer}

The values for dynamic viscosity of the glycerol solutions determined from a stress-step experiment show good agreement with the theoretical values for concentrations up to $70 \%$ of glycerol. At the same time, a slip detection method employed in the previous study allowed for accurate

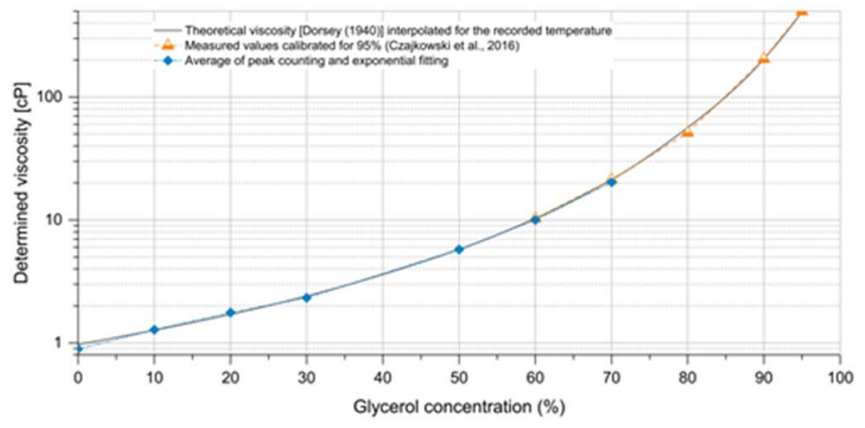

Fig. 6. A complete range of glycerol viscosities is measurable using the developed device.

measurements when the concentration of glycerol was greater than $60 \%$. Therefore, we can effectively combine two probing modalities as shown in Fig. 6, where we can observe a good agreement between the experimental and theoretical viscosity values across the studied concentration range. Consequently, we are able to significantly extend the dynamic range of the device from previously reported 10 $400 \mathrm{cP}$ to $\sim 1.1-400 \mathrm{cP}$ in dual detection mode.

In our study we have developed a novel approach to phaseslip detection based on altering the magnitude of the driving magnetic field while its frequency is kept constant. The dynamic viscosity is then proportional to the amplitude of current, for which the phase slipping occurs. The applied rate of rotation can be optimized for a particular measurement scenario in order to minimize the unwanted friction and inertia-related effects. As indicated in our previous work, the device is still in prototype phase and therefore has some deficiencies [18]. When using a field angle sensor, the requirements for components placement is high to avoid any non-linearities to angular response of the device.

What comes from the theoretical treatment presented by Baravian \& Quemada [22], the ringing frequency depends on the instrumental parameters and sample elasticity as $\omega$ ? $\sqrt{b G^{\prime} / I}$. Therefore, expressed by Ewoldt, this frequency can be improved by increasing $\sqrt{b / I}$. If the inertia of the geometry is much less than the inertia of the instrument, then the choice of geometry should be made to increase $b$ [23]. Consequently, increasing the magnetization of the probe should minimize the distortions and improve the accuracy of the angular sensing. However, the mass and the size of a magnet should be minimized to avoid excess inertia of the instrument. The probe design and a magnet should be carefully optimized to improve the sensitivity without increasing its inertia.

TABLE I. SUMMARY OF THE TRACEABILITY MEASUREMENTS

\begin{tabular}{|l|c|c|c|c|c|c|}
\hline Test fluid & $\begin{array}{c}\text { Viscous } \\
\text { component }\end{array}$ & $\begin{array}{c}\text { Error of viscous } \\
\text { component [\%] }\end{array}$ & Inertia & $\begin{array}{c}\text { Error of inertia } \\
\text { [\%] }\end{array}$ & Final orientation & $\begin{array}{c}\text { Error in } \\
\text { orientation [\%] }\end{array}$ \\
\hline RT5 & 4.994 & 1.54 & 6.551 & 1.01 & 80.658 & 0.84 \\
\hline RT10 & 9.756 & 0.89 & 6.728 & 1.03 & 74.786 & 0.68 \\
\hline RT50 & 55.233 & 5.85 & 9.173 & 11.48 & 40.893 & 0.75 \\
\hline RT100 & 124.188 & 1.80 & 36.866 & 4.39 & 41.343 & 0.63 \\
\hline RT250 & 329.821 & 1.77 & 265.015 & 5.50 & 41.814 & 1.50 \\
\hline
\end{tabular}




\section{E. Traceability measurements}

In order to understand better the metrologically relevant accuracy of the proposed rheometer, the calibrated liquids at particular viscosity (RT5, RT10, RT50, RT100 and RT250) from Paragon Scientific Ltd was tested. The summary of the obtained rheological parameters with error estimation is shown in Table I.

\section{CONCLUSION}

The proposed dual detection method was shown experimentally to be capable to extend the dynamic range of the magnetic sensing and actuation based rheometer especially in the range of low viscosities (below $10 \mathrm{cP}$ ). Improved viscosity measurement range combined with other advantageous proporties of the concept (disposalbe probe, fast detection, measurements in hermetic conditions, parallel measurements) makes it more applicable for different applications. In this paper, the viscosity measurements were shown to work for fluids between $1.1-400 \mathrm{cP}$ but optimizing the probe design, the measurement range is expected to be extended for much higher viscosities as well. This is expected to lead new solutions to existing problems in the challenging sensing applications where volatile and flammalbe fluids are measured or where the risk of contamination is a major concern.

\section{REFERENCES}

[1] J.D. Ferry, "Viscoelastic properties of polymer solutions," J. Res. Nat. Bur. Stand., vol.41, pp. 53-61, 1948.

[2] A. P. Nowak et al., "Rapidly recovering hydrogel scaffolds from selfassembling diblock copolypeptide amphiphiles," Nature, vol. 417, pp. 424-428, 2002.

[3] T. M. Squires, T.G. Mason, "Fluid mechanics of microrheology," Annu. Rev. Fluid Mech., vol. 42, pp. 413-438, 2010.

[4] T. A. Waigh, "Microrheology of complex fluids,". Rep. Progr. Phys., vol. 68 , pp. $685,2005$.

[5] C. Baravian, G. Benbelkacem, F. Caton, "Unsteady rheometry: can we characterize weak gels with a controlled stress rheometer?," Rheol. Acta, vol. 46, pp. 577-581, 2007.

[6] G. G. Fuller, Optical Rheometry of Complex Fluids. Oxford Univ. Press, New York, 1995.

[7] H. J. Walls, R. F. Berg, E. J. Amis, "Multi-sample couette viscometer for polymer formulations," Meas. Sci. Technol. vol. 16, pp. 137-137, 2005.

[8] S. Dennington, et al., "Miniaturized rotating disc rheometer test for rapid screening of drag reducing marine coatings," Surface Topography: Metrology and Properties, vol. 3, 034004, 2015

[9] A. Tokarev, et al., "Multifunctional magnetic rotator for micro and nanorheological studies,” Rev. Sci. Instrum., vol. 83, pp. 1-8, 2012.

[10] A. Tokarev, et al., "Probing viscosity of nanoliter droplets of butterfly saliva by magnetic rotational spectroscopy," Appl. Phys. Lett., vol. 102, no. 3,033701, 2013.

[11] K. Fukunaga, et al., "Blood viscometer applying electromagnetically spinning method," Int. J. Artif. Organs, vol. 16, pp. 359-367, 2013.

[12] I. Sinn, et al., "Asynchronous magnetic bead rotation microviscometer for rapid, sensitive, and label-free studies of bacterial growth and drug sensitivity," Anal. Chem., vol. 84, pp. 5250-5256, 2012.
[13] L. Pavlovsky, J. G. Younger, M. J. Solomon, "In situ rheology of staphylococcus epidermidis bacterial biofilms," Soft. Matter., vol. 9, pp. 122-131, 2013.

[14] P. Kinnunen, et al., "Rapid bacterial growth and antimicrobial response using self-assembled magnetic bead sensors," Sens. Actuators B, vol. 190, pp. 265-269, 2014.

[15] B. H. McNaughton, K. A. Kehbein, J. N. Anker, R. Kopelman, "Sudden breakdown in linear response of a rotationally driven magnetic microparticle and application to physical and chemical microsensing," J. Phys. Chem. B, vol. 110, pp. 18958-18964, 2006.

[16] R. McKennell, "Cone-plate viscometer," Anal. Chem., vol. 28, pp. 1710-1714, 1956.

[17] S. de Lucena, W. Kaiser, "Stepping motor driven constant-shear-rate rotating viscometer," IEEE Trans. Instrum. Meas. vol. 57, pp. 13381343, 2008.

[18] J. Czajkowski, et al. "Simultaneous magnetic actuation and observation with ferromagnetic sensors," Meas. Sci. Technol., vol. 2, 025301, 2016.

[19] L. Chevry, N. K. Sampathkumar, A. Cebers, J. -F. Berret, "Magnetic wire-based sensors for the microrheology of complex fluids," Phys. Rev. E, vol. 3, 062306, 2013.

[20] K. Haapanen, et al., "Observation of viscoelastic solutions with ferromagnetic stirrers," Sens. Actuators A, vol. 236, pp. 309-314, 2015.

[21] G. Helgesen, P. Pieranski, A. T. Skjeltorp, "Dynamic behavior of simple magnetic hole systems," Phys. Rev. A, vol. 42, pp. 7271-7280, 1990.

[22] C. Baravian, D. Quemada D., "Using instrumental inertia in controlled stress rheometry", Rheol. Acta, vol. 37, pp. 223-233, 1998.

[23] R. H. Ewoldt, G. H. McKinley, "Creep ringing in rheometry or how to deal with oft-discarded data in step stress tests!," Rheol. Bull., vol. 76, 2007.

[24] H. A. Barnes, D. Bell, "Controlled-stress rotational rheometry: An historical review," Korea-Australia Rheol. J., vol. 15, pp. 187-196, 2003.

[25] R. Roscoe, "Free damped oscillations in viscoelastic materials," J. Phys. D. Appl. Phys., vol. 2, 1261, 1969.

[26] G. Benmouffok-Benbelkacem et al., "Non-linear viscoelasticity and temporal behavior of typical yield stress fluids: Carbopol, xanthan and ketchup," Rheol. Acta, vol. 49, pp. 305-314, 2010.

[27] A. Jaishankar, V. Sharma, G. H. McKinley, "Interfacial viscoelasticity, yielding and creep ringing of globular protein-surfactant mixtures," Soft. Matter., vol. 7, pp. 7623-7634, 2011.

[28] L. Struik, "Free damped vibrations of linear viscoelastic materials," Rheol. Acta, vol. 6, pp. 119-129, 1967.

[29] R. Roscoe, "Free damped oscillations in viscoelastic materials," J. Phys. D. Appl. Phys., vol. 2, 1261, 1969.

[30] I. M. Krieger, "Bingham award lecture-1989: The role of instrument inertia in controlled-stress rheometers," J. Rheol., vol. 34, pp. 471-483, 1990.

[31] M. Mackay, V. Liang, P. Halley, "Instrument effects on stress jump measurements," Rheol. Acta, vol. 31, pp. 481-489, 1992.

[32] U. Zölzer, H.F. Eicke, "Free oscillatory shear measurements - an interesting application of constant stress rheometers in the creep mode," Rheol Acta, vol. 32, pp. 104-107, 1993.

[33] A. Jaishankar, G. H. McKinley, "Power-law rheology in the bulk and at the interface: quasi-properties and fractional constitutive equations," Proc. R. Soc. London, Ser. A, vol. 469, 2012.

[34] F. Mainardi, G. Spada, "Creep, relaxation and viscosity properties for basic fractional models in rheology,” Eur. Phys. J. Spec. Top., vol. 193, pp. 133-160, 2011.

[35] N. E. Dorsey, "Properties of ordinary water-substance, in all its phases: water vapor, water, and all the ices," Monograph series, American Chemical Society, vol. 81, pp. 182-184, 1940. 